\title{
ESTRATEGIAS DE ARMADO EN ESTRUCTURAS RETICULADAS DE HORMIGON
}

\author{
(REINFORCEMENT STRATEGIES IN RETICULATED CONCRETE STRUCTURES)
}

Ricardo Aroca Hernández-Ros, Dr. Arquitecto

Catedrático de la E. T. S. de Arquitectura de Madrid/España
Fecha de recepción: 31-XI-88

\section{RESUMEN}

La influencia de la cuantía y disposición de armaduras en la redistribución de momentos flectores en una pieza de hormigón armado, trae como consecuencia que la comprobación o la determinación de la seguridad de una estructura se realice por un camino "inverso":

1) Determinación de una estrategia de armado.

2) Comprobación de la ductilidad de la estructura y de la distribución real de momentos. 3) Determinación del margen de seguridad en esa sección.

\section{SUMMARY}

The effect of the quantity and arrangement of reinforcements in the redistribution of bending moments in a piece of reinforced concrete means that the testing or determination of the safeness of a structure is carried out inversely: 1) Determining a reinforcement strategy; 2) Testing the ductility of the structure and the real arrangement of moments; 3) Determining the safety margen of said section.
La práctica habitual de armar las piezas de hormigón de una forma estricta para los puntos de momento máximo produce piezas de rigidez variable a flexión (rigidez que tiene más que ver con el canto y la cuantía de armado, que con la inercia de la sección del hormigón).

La simple consideración del papel de la armadura en la rigidez local lleva, aún en periodo elástico, a acentuar las puntas de momentos y exige (salvo para algunos casos de carga) que, para que piezas flectadas tengan realmente la seguridad calculada, se produzcan unos giros plásticos antes de la rotura.

La comprobación de ductilidad se revela, en consecuencia, como el factor más importante en la determinación de la seguridad real de la estructura.

No es posible el cálculo de la distribución real de mo- mentos, ni de la ductilidad necesaria sin una determinación previa de las armaduras, para lo que es preciso formular estrategias previas de armado.

De hecho si la estretegia de armado asegura:

a) Un esquema de solicitaciones en equilibrio con el sistema de acciones.

b) Una resistencia en cada sección, al menos igual a la solicitación prevista en el esquema de equilibrio.

La comprobación puede reducirse a la de si la ductilidad de algunos puntos de la estructura es suficiente.

El seguir el método convencional de cálculo de solicitaciones previo al armado puede, en algunos casos, conducir a estructuras con menor margen de seguridad del calculado por falta de la necesaria ductilidad. 


\section{Giros necesarios y posibles}

La expresión de la curvatura local de una viga de hormigón armado es:

$\frac{1}{\mathrm{R}}=\frac{\epsilon_{\mathrm{c}}+\epsilon_{\mathrm{s}}}{\mathrm{d}}=\epsilon_{\mathrm{s}} \frac{\mathrm{C}}{\mathrm{d}} ;$ siendo $\mathrm{C}=1+\frac{\epsilon_{\mathrm{c}}}{\epsilon_{\mathrm{s}}} ;$

Siendo e la cuantía geométrica de la armadura de tracción:

$$
C=\frac{1}{1-\sqrt{\left(E_{s} / E_{c}\right)^{2} \varrho^{2}+2\left(E_{s} / E_{c}\right)} \varrho-\left(E_{s} / E_{c}\right) \varrho}
$$

Mientras las deformaciones del hormigón se mantengan dentro de un grado razonable de proporcionalidad con las tensiones; por lo que la curvatura local de una pieza solicitada por un determinado momento flector dependerá de:

- La deformación unitaria de la armadura de tracción.

- El canto útil de la pieza.

- La relación de módulos de elasticidad acero/hormigón.

- La cuantía geométrica de la armadura.

La cuantía de la armadura y el canto de la pieza, y no la inercia de la envolvente de hormigón, son los factores determinantes en la curvatura local y, por tanto, en la rigidez y deformación de la viga.

En las condiciones de proporcionalidad, antes postulados, no resulta difícil mediante una simple aplicación de los teoremas de Mohr hallar los momentos de extremo de una viga doblemente empotrada, sometida a cualquier esquema de carga.

Admitiendo 3 zonas de armado, con sus correspondientes relaciones momento/curvatura, expresado por diferentes valores de $\mathrm{C}: \mathrm{C} 1, \mathrm{CO}$ y $\mathrm{C} 2$.

Supuestos unos momentos de extremo $M 1$ y $M 2$, basta, partiendo de cada uno de los 2 extremos, comprobar que el otro coincide con el teórico punto de apoyo para determinar la validez de la hipótesis.

$$
\begin{aligned}
& \frac{\delta_{2}}{L}=\frac{1}{L} \int_{0}^{L} \frac{1}{R}(L \cdot x) d x=\lambda \int_{0}^{1} \epsilon_{s} C(1 \cdot x / L) \cdot d(x / L) \\
& \text { siendo } \lambda=\frac{L}{d} \\
& \frac{\delta_{1}}{L}=\lambda \int_{0}^{1} \epsilon_{s} C(1 \cdot x / L) \cdot d(x / L)
\end{aligned}
$$
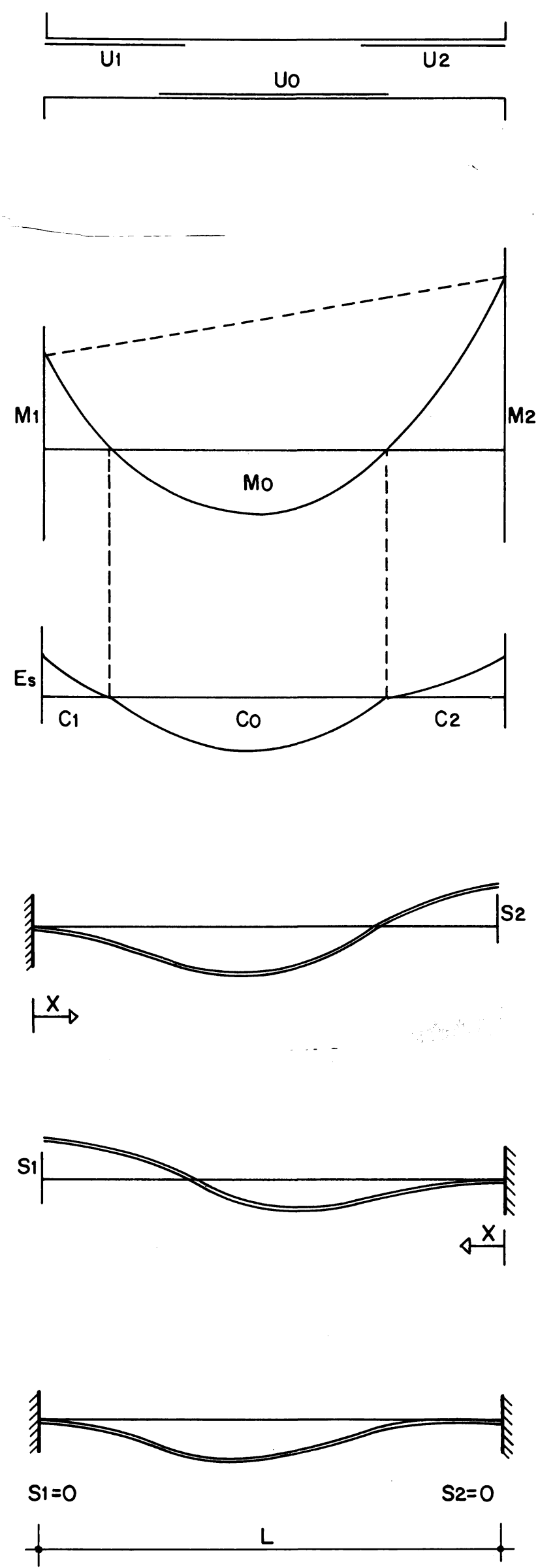
Los valores reales de $M 1$ y $M 2$ serán los que cumplan la condición $\delta_{1}=\delta_{2}=0$.

Si para $\delta_{1}=\delta_{2}=0$ ninguno de los tres momentos M0, M1 y $M 2$ superan el límite en que empiezan a producirse deformaciones no proporcionales, la solución al problema es correcta teniendo en cuenta que MO, M1 y M2 están relacionados por la gráfica conocida de momentos isostáticos; las dos condiciones de borde son suficientes para determinar la gráfica de momentos real de la pieza, bien mediante el planteamiento de ecuaciones, bien por aproximaciones sucesivas.

Aplicando lo antes expuesto a una pieza con carga uniforme y ambos extremos impedidos de giro, para distintas distribuciones de una armadura total $U$, obtenemos los siguientes resultados:

Para obtener una correspondencia entre la distribución de momentos y la armadura deberíamos recurrir a esquemas de armados muy lejanos de los sancionados por la experiencia. La alternativa no es otra que admitir que deben producirse unas redistribuciones plásticas y que para cargas repartidas estas redistribuciones se producirán en los extremos de las vigas, en los que la relación momento último/momento real es mucho menor que en el acto.

Si consideramos por una parte la deformación que adquirirá la viga de continuar el comportamiento lineal momento/curvatura para deformaciones mayores del hormigón, y por otro los giros plásticos que serán debidos a dos factores: las deformaciones no proporcionales del hormigón y las deformaciones plásticas del acero. (Los giros plásticos estarán bastante localizados, siendo la hipótesis lineal válida para la mayor parte de la pieza).

Como por otra parte, en un esquema de carga creciente proporcionalmente y asumiendo una suficiente ductilidad de los extremos de la pieza, la situación de rotura se alcanzará para una línea de cierre conocida en función de la distribución de armaduras:

Por aplicación de las fórmulas anteriores podemos conocer la deformada final de la viga, así como los giros plásticos necesarios en los extremos 1 y 2. (Si uno de los valores, 1 ó 2, tuviera signo contrario, ello significaría que una de las rótulas se produciría en la zona central de la viga).
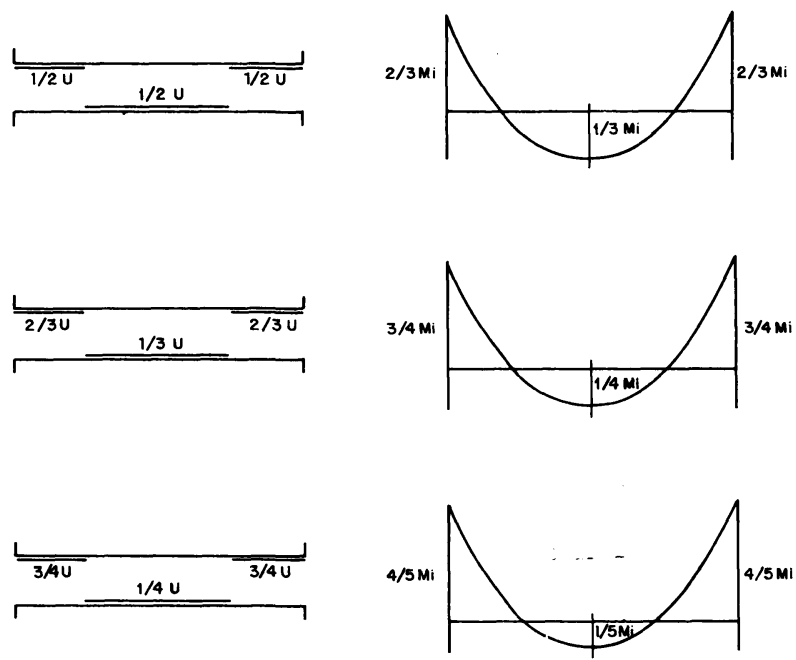
El proceso de diseño/comprobación de una pieza puede pues descomponerse fácilmente en varias partes:

1. $\left.{ }^{\circ}\right)$ Fijación de las armaduras U1, U2 y U0 (estrategia de armado).

2.) Determinación de los momentos últimos correspondientes, que a su vez permiten conocer el momento isostático Mi y, por lo tanto, la carga última.

3. ) Determinación de los giros plásticos necesarios.

4. $\left.{ }^{\circ}\right)$ Comprobación de que los giros plásticos necesa. rios son menores que los posibles.

Como este artículo está orientado hacia las estrategias de armado (punto 1) no se entra en el punto $4 .^{\circ}$, puesto que el argumento es compatible con las distintas hipótesis que pueden formularse al respecto.

La magnitud de los giros necesarios para justificar el comportamiento de una viga de H.A. hace preciso que una longitud finita de la armadura adquiera una deformación plástica apreciable, lo que a su vez supone que la curvatura local sea compatible con la existencia de una zona comprimida de hormigón suficiente para mantener el equilibrio.

Mientras que en zonas de tangente horizontal a la gráfica de momentos no es difícil imaginar el funcionamiento de la rótula, en los extremos y en general en puntos de gráfica de momentos correspondientes es necesario recurrir a un esquema de bielas para explicar la posibilidad de formación de una rótula plástica (una manera de que longitud finita de armadura plastificada sea compatible con momento posible).

Sea cual fuere el esquema de rótula que se adopte, el giro posible depende fundamentalmente de la profundidad de la cabeza comprimida de hormigón y, por tanto, la diferencia de cuantías mecánicas entre armadura extendida y comprimida.

$\gamma=\frac{\mathrm{U} \cdot \mathrm{U}^{\prime}}{\text { bdfc }}$, mientras el giro necesario depende fuertemente de la esbeltez de la pieza.
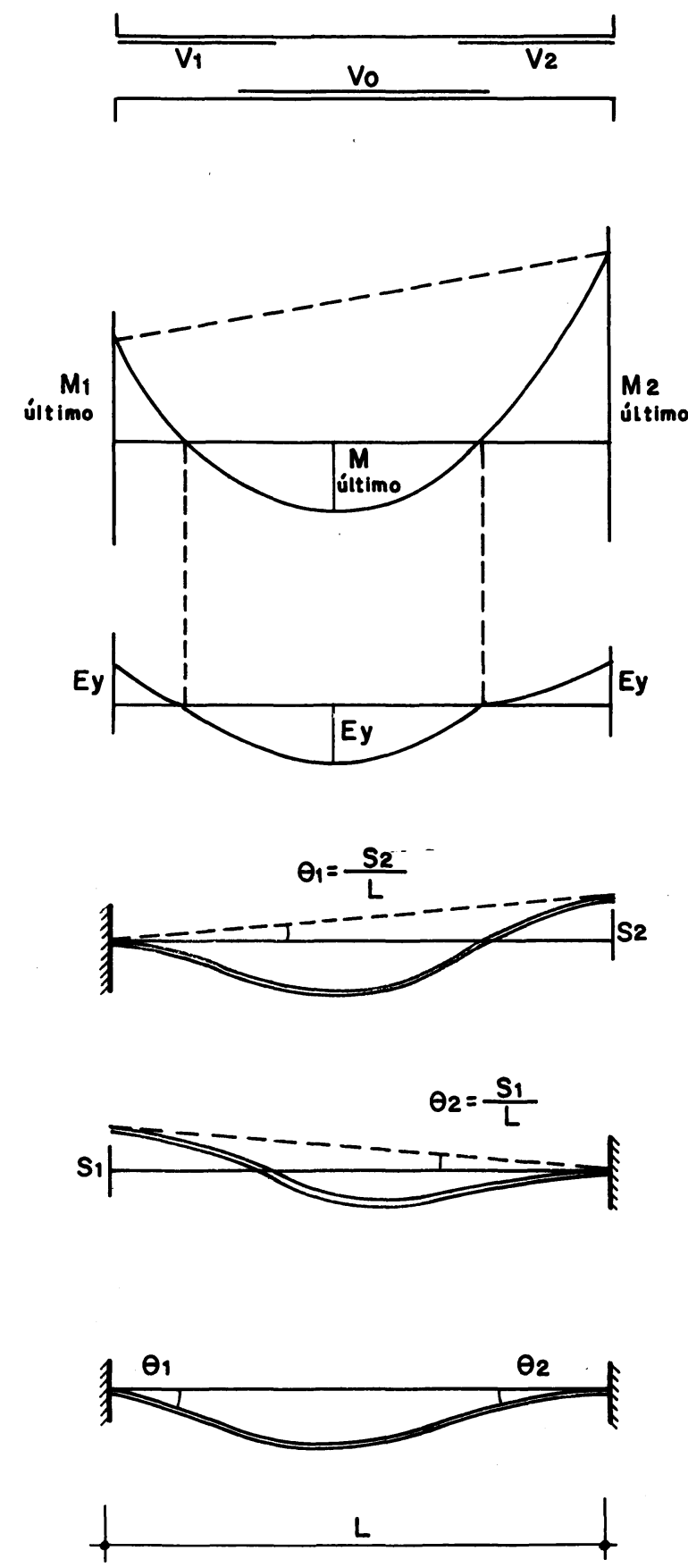

http://informesdelaconstruccion.revistas.csic.es 
Tendremos, pues, unos giros necesarios con expresiones del tipo:

$\theta=\frac{\delta}{\mathrm{L}} \lambda \cdot \epsilon_{\mathrm{y}} \frac{\epsilon_{\mathrm{s}}}{\epsilon_{\mathrm{y}}} \mathrm{C}(1-\mathrm{x} / \mathrm{L}) \mathrm{de}(\mathrm{x} / \mathrm{L})$

El valor de la expresión recuadrada depende solamente de la forma de la gráfica $\in_{\mathrm{s}} / \in_{\mathrm{y}}$. En las que sólo hay 2 puntos variables para un tipo de carga dado en función de los paramentos de: cuantías geométricas de las armaduras y relación de módulos de elasticidad.

Siendo los factores más importantes la esbeltez $\lambda$ y el limite elástico de acero $\epsilon_{y}$ :

La expresión $\int_{0}^{1} \frac{\epsilon_{s}}{\epsilon_{y}} C(1-x / L) d(x / 1)$, alcanzará valores

más altos para armados U1 y U2 pequeños, y valores pequeños para valores altos de armados de extremo.

De hecho no es más que el momento estático de las áreas de las figuras respecto a los extremos, multiplicando cada una de ellas por el correspondiente valor de $\mathrm{C}$.

Así, pues, a igualdad de tipo de acero y esbeltez, los armados de extremos débiles corresponderán a giros necesarios altos, mientras que los armados altos corresponden a giros nécesarios pequeños.

Recordando que los giros posibles son, por otra parte, función de la cuantía relativa de armadura, cabe concluir que implícitamente existe una posibilidad de compensación. Para armados débiles de extremo son a la vez necesarios y posibles giros mayores, mientras que para armados fuertes son necesarios y posibles giros menores.

La frontera exacta es difícil de determinar hasta reunir evidencia experimental. Para ajustar los paramentos del modelo, no obstante es posible hacer algunas afirmaciones de tipo indicativo:

1) Cuanto más esbelta es una pieza, más probable es que el giro necesario exceda el posible y, por tanto, sea menos segura de lo calculado en un proceso estándar.

2) Las cuantias moderadas de armado y/o la colocación de armaduras de compresión aumentan a ductilidad y hacen menos probable una rotura con menor carga que la prevista.
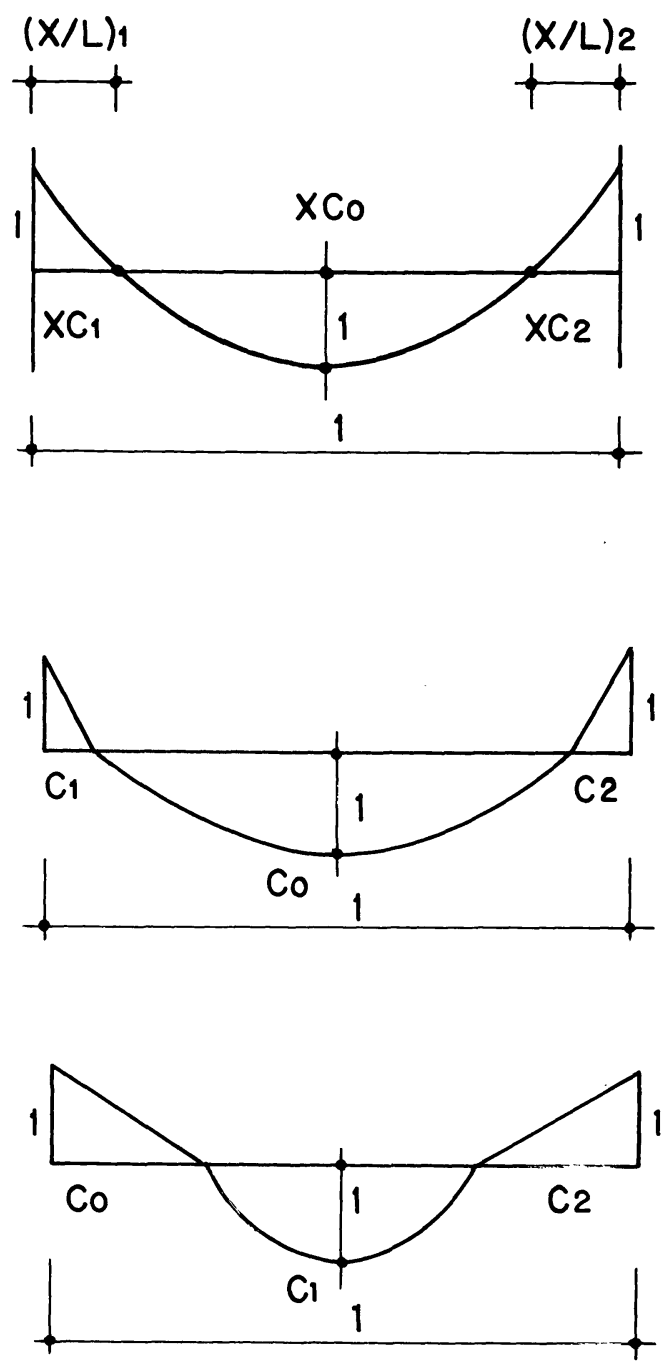

3) El empleo de aceros de más alto límite elástico incrementa los giros necesarios reduciendo la posibilidad de que cuadre el balance de giros de la pieza.

En consecuencia, caso de emplear vigas esbeltas y momentos de extremo próximos al de agotamiento es conveniente colocar armaduras de compresión en un factor creciente con la esbeltez de la pieza.

Suponiendo que disponemos de un criterio fiable para colocar suficiente armadura de compresión en función de la esbeltez de la viga, las cuantías de armado y el tipo de acero (hay que señalar que la necesidad de estos criterios es la misma aun cuando se diseñe la estructura por procedimientos "elásticos") pueden establecer una relación inmediata entre los momentos isostáticos y las armaduras de la pieza (de hecho el brazo de ponderado varía muy poco del correspondiente a un momento Mi/2), de manera que conocido Mi puede hallarse la armadura total $\mathrm{U}$. 
Para el caso de que se piense disponer armaduras distintas en los dos extremos, puede ayudar a establecer una relación entre momento isostático y armaduras:

$M o=M i \cdot \frac{M 1+M 2}{2}+M i \frac{\triangle^{2}}{4}$

Lo que traducido a armaduras

$\mathrm{Uo}_{\mathrm{o}}=\mathrm{U} \cdot \frac{\mathrm{U} 1+\mathrm{U} 2}{2}+\mathrm{U} \cdot \frac{\triangle^{2}}{4}$ siendo $\triangle=\frac{\mathrm{U} 1 \cdot \mathrm{U} 2}{2 \mathrm{U}}$

El término $U \cdot \triangle^{2} / 4$ define el aumento de armado global debido a la desigualdad de las armaduras de extremo.

\section{Estrategias de armado}

De las simulaciones realizadas se reduce la existencia de una banda de distribución de armaduras posibles, coincidentes por otra parte con las empleadas en la práctica habitual, en que la resistencia y rigidez de las vigas resulta prácticamente indistinguibles.

En el caso de piezas de esbeltez 20 y superiores resulta necesario prolongar las armaduras de pórticos (e incluso incrementarlas) hasta el apoyo.

\section{Vigas continuas}

La estrategia de diseño es la típica del cálculo plástico y puede dibujarse arbitrariamente la línea de cierre (excepto en el caso de los voladizos).

Parece razonable buscar un trazado que deje unos puntos de momento del orden de $1 / 2$ a 2/3 de los momentos isostáticos.

La sección de hormigón debe ser suficiente para que las cuantías mecánicas para un momento mitad del isostático máximo no sean superiores a 0,$3 ;$ y si la viga es esbelta debe comprobarse la posibilidad de giro en las rótulas plásticas que se formarán en los apoyos y calcular que existe suficiente armadura de compresión.

Para esbelteces altas es conveniente prolongar y anclar en el apoyo la totalidad de la armadura de positivos y acercar la relación de armaduras al 2/1, con objeto de reducir la necesidad de rotaciones plásticas.
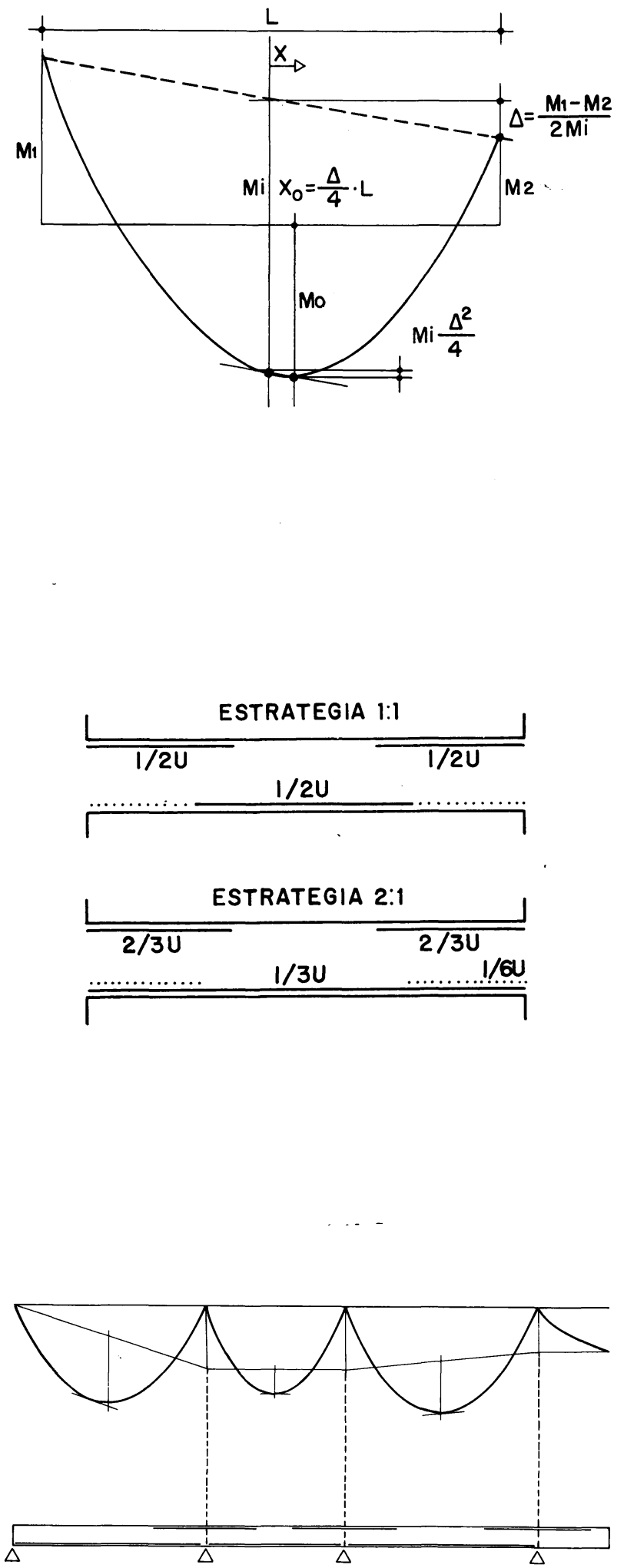

Dado que regulamos a voluntad la resistencia de la viga en cada punto, podemos asegurar que en un proceso gradual de carga el colapso se producirá de forma prácticamente simultánea en todos los tramos, es decir, la seguridad a rotura es uniforme. 


\section{Pórticos con cargas verticales}

La manera más sencilla de colocar la armadura de momentos negativos implica su continuidad en tramos contiguos, lo que lleva a que en situación de colapso se produzca una rótula a cada lado del soporte y éste quede sometido a compresión simple.

En un proceso de carga progresiva el mayor momento en el soporte se produce para una carga entre el 70 por ciento y el 80 por 100 de la carga de rotura.

En la determinación de momentos en los soportes aparecen factores contrapuestos:

- Por un lado, los momentos desequilibrados al aproximarse la carga de las vigas a la de colpaso disminuyen, llegando a anularse.

- Por el otro, las vigas reales tienen menos rigidez de la correspondiente a la inercia de la envolvente de hormigón y los soportes más, por lo que en un proceso de carga gradual los momentos iniciales en los soportes son los correspondientes a un análisis convencional.

Colocándonos del lado de la seguridad podriamos hacer una doble comprobación de los soportes:

- A compresión simple para la carga última.

- A flexión compuesta con un 75 por 100 de la carga última y el total momento desequilibrado de las vigas (lo que está del lado de la seguridad).

Para soportes interiores, la primera comprobación incluye a la segunda; en caso de vigas del mismo canto y carga uniforme si

$$
\frac{L 1-L 2}{1,6 n}<d
$$

siendo "L1" la luz mayor, "L2" la menor " $n$ " el número de plantas sobre la que se está estudiando y "d" el canto del soporte.

Cuando en el soporte confluyen vigas de distinto canto, no desaparecerá el momento desequilibrado, que será la diferencia de momentos últimos de las vigas.

En el caso de los soportes extremos, el momento del soporte se elige al determinar la armadura de la viga.

En el soporte superior, el momento será el de la viga, y en los intermedios la mitad aproximadamente (colocando la misma armadura de negativos en ambos lados del pórtico se evitan cortantes horizontales desequilibradas en el momento del colapso).
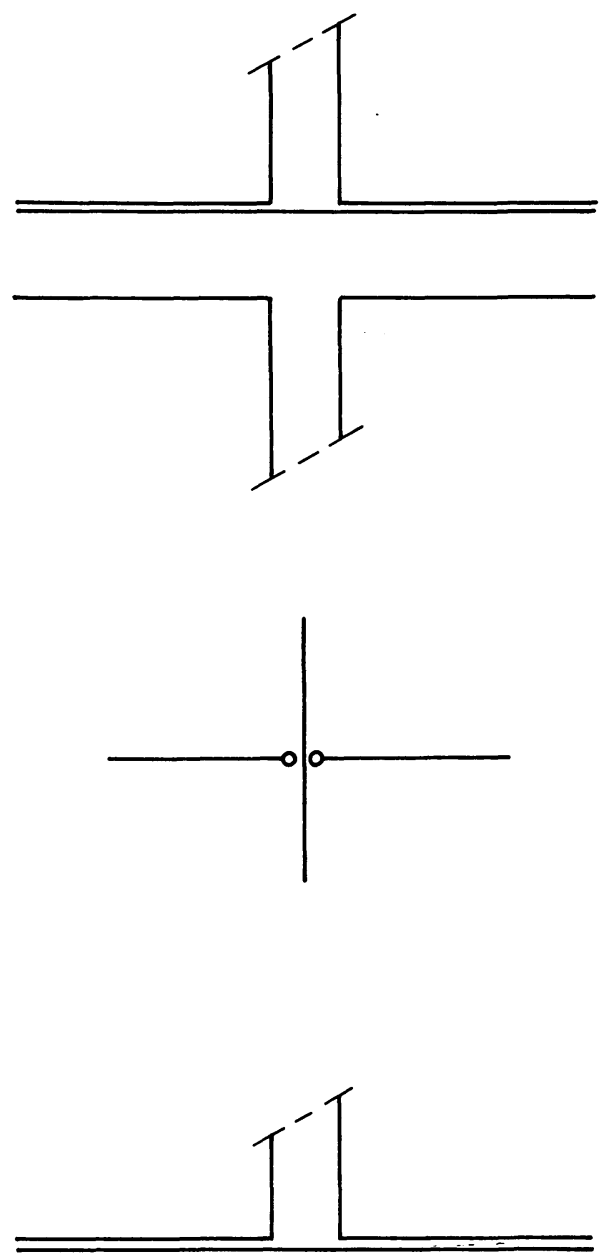

U1y2 


\section{Resumiendo:}

Las vigas pueden armarse como vigas continuas con unos armados totales por tramo que cubran el momento isostático, procurando que los puntos de momento se encuentren entre $1 / 2$ y $2 / 3$ de $\mathrm{Mi}$; los momentos de extremo pueden elegirse arbitrariamente, aunque teniendo en cuenta la rigidez de los soportes.

Los soportes interiores, con las limitaciones antes descritas, pueden dimensionarse a compresión simple para la carga última.

Esta estrategia conduce a un cierto ahorro respecto a los procedimientos convencionales, ya que la desaparición de momentos desequilibrados en los nudos permite armar algo menos, tanto las vigas como los soportes.

\section{Estabilidad}

Con un dimensionado estricto, el pórtico se convierte en un mecanismo, de manera que si no hay otros medios de garantizar la estabilidad, es preciso asegurar que en cada planta haya al menos una cruz con armado sobredimensionado, de manera que no se produzcan rótulas y se pueda asegurar respuesta suficiente para restablecer la verticalidad del conjunto.

Dada la necesidad de tener en cuenta las acciones horizontales no merece la pena desarrollar este apartado.

\section{Combinación de acciones horizontales y verticales: mecanismos}

Suponiendo un proceso de carga creciente, en el que se mantenga la proporción entre acciones verticales y horizontales, parece razonable diseñar el pórtico para que se produzca un mecanismo de rotura como el de la figura, de manera que todas las rótulas estén en las vigas $y$, a ser posible, se produzcan al mismo tiempo.

En cada tramo aparecen dos articulaciones, una en el extremo de sotavento y la otra en el vano.
El equilibrio global del pórtico implica que:

$\Sigma T s=W$

$\Sigma T V \cdot L=W \cdot h$

Los valores individuales de Ts y Tv dependerán de la geometría, dimensiones y estrategia de armado.

Supongamos el problema resuelto; en cada tramo de viga tendremos un momento isostático "Mi" debido a las cargas verticales y un momento debido a la acción horizontal:

$\mathrm{Mw}=\frac{\mathrm{Tv} \cdot \mathrm{L}}{2}$
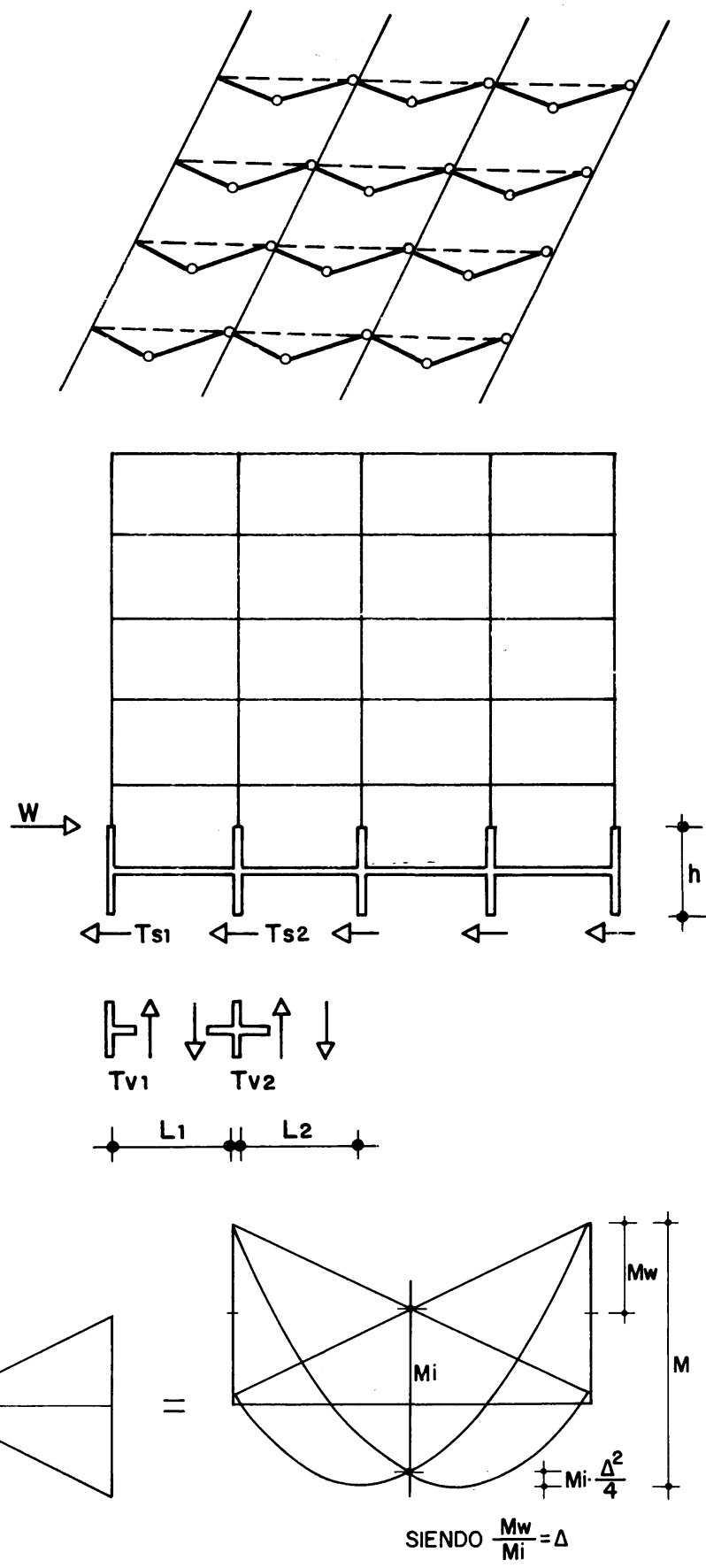
Es fácil calcular, tanto la contribución de la viga a la inclinación del pórtico antes de la aparición de la segunda rótula, $\theta_{\mathrm{v}}$, como el giro plástico necesario en el extremo de sotavento $\theta_{2}$.

La compatibilidad de deformaciones requiere que la inclinación sea constante en cada planta, y corresponderá a la del tramo de menor rigidez, lo que obligará a que los demás tramos desarrollen la segunda rótula y sigan adquiriendo deformación plástica y, por tanto, aumentando el giro necesario.

Si es $\triangle \theta=\theta_{v}$ máx $-\theta_{v}$

$$
\triangle \theta_{2}=\Delta \theta \cdot \frac{\mathrm{X}_{0}}{\mathrm{~L} \cdot \mathrm{X}_{\mathrm{o}}}
$$
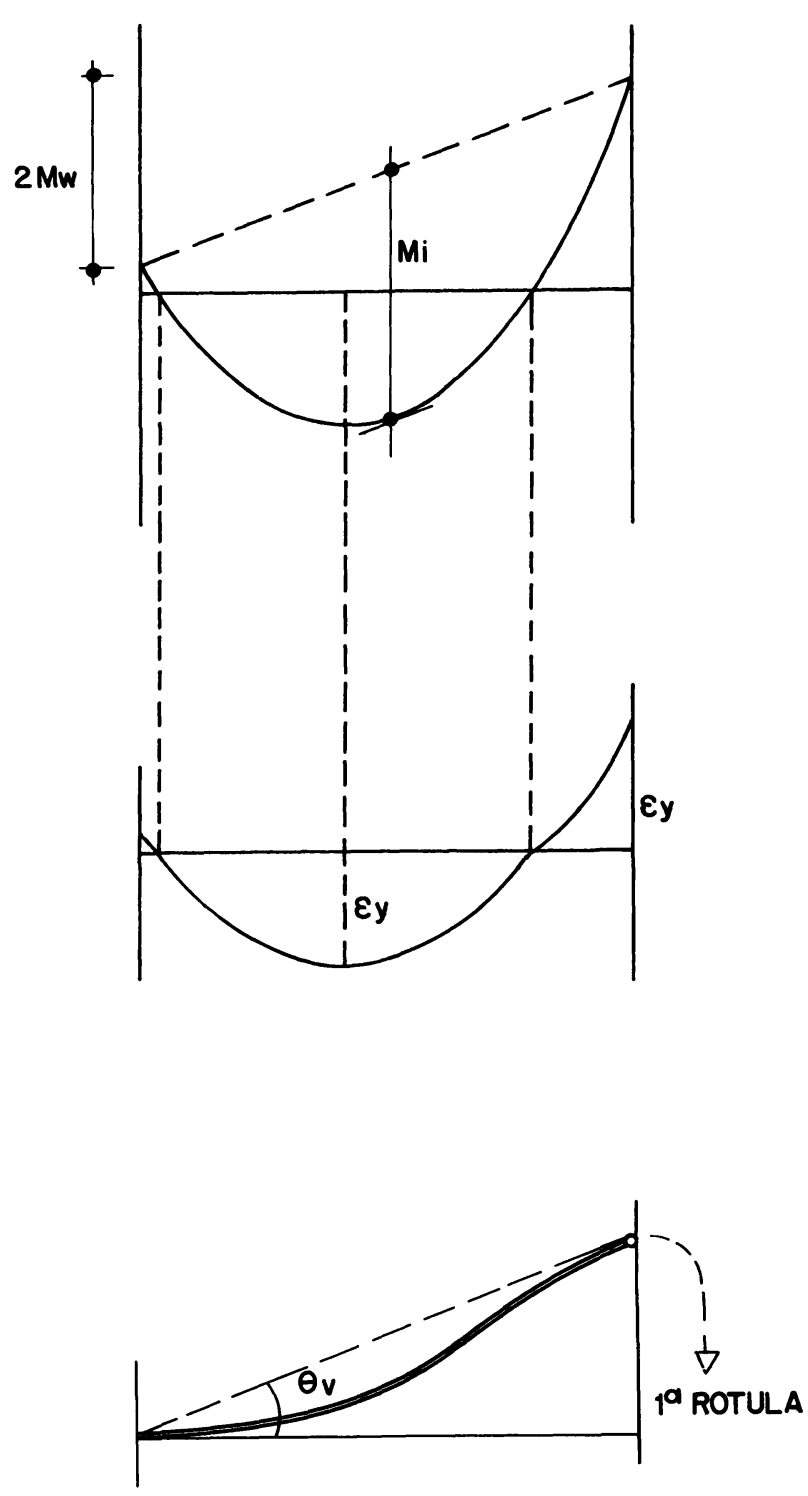

es el aumento de giro necesario en la rótula 2

$$
\theta_{0}=\Delta \theta+\Delta \theta_{2}=\Delta \theta \frac{\mathrm{L}}{\mathrm{L} \cdot \mathrm{X}_{\mathrm{o}}}
$$

Lo deseable es que los $\theta_{v}$ sean semejantes en todos los tramos y, además, tengan un valor limitado de manera que el efecto de segundo orden no aumente excesivamente los momentos.

Dimensionando los soportes, de manera que no se produzcan rótulas en ellos, su contribución a la inclinación general del pórtico será mínima.
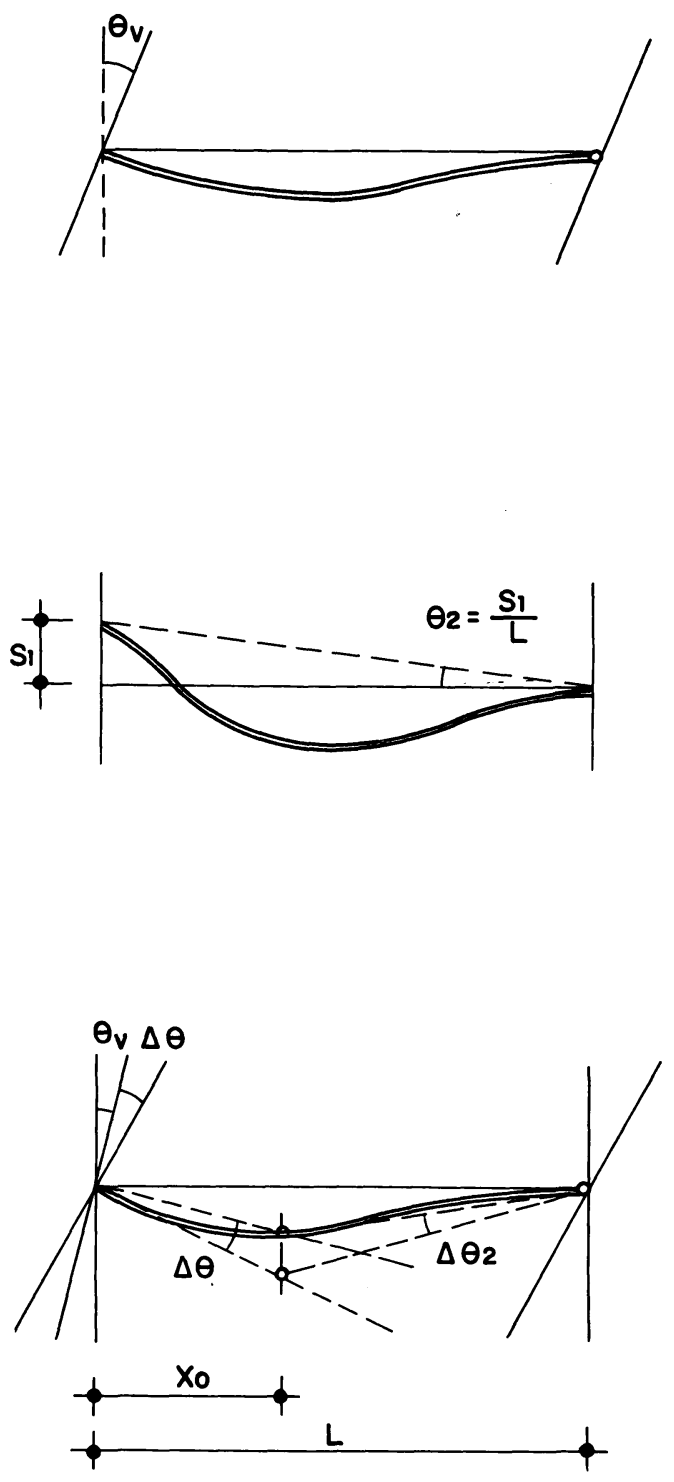

http://informesdelaconstruccion.revistas.csic.es 


\section{Estrategias de diseño}

Si no hay limitación del canto de las vigas - una decisión razonable cuando es importante - la acción horizontal es dimensionar las vigas con esbeltez constante; esto tiene tres ventajas:

a) La rigidez de todos los tramos es sensiblemente igual, por lo que las inclinaciones asociadas a cada tramo serán semejantes.

b) Al ser común la armadura de momentos negativos de dos tramos contiguos, los momentos últimos serán sensiblemente proporcionales a los cantos.

Un aumento de armadura, sobre la necesaria, para resistir la acción gravitatoria permitirá que cada tramo pueda resistir un excedente de momento proporcional a su canto y, por lo tanto, a su luz, de manera que las líneas de cierre bajo la acción combinada última tendrán la misma pendiente, es decir, el cortante hiperestático de cada tramo será el mismo y nos encontraremos con la misma situación de tramos de igual luz; solamente habrá variaciones de normal en los soportes extremos por la acción horizontal y el pórtico funcionará con la máxima eficacia.

c) Dada la expresión de la componente de la inclinación, debida a la deformación de las vigas, si $\lambda$ es constante, los valores de $\theta_{v}$ serán prácticamente iguales en todos los tramos, por lo que el pórtico tendrá la mínima inclinación posible.

Este criterio de diseño deberá emplearse razonablemente cuando $\mathrm{Mw}$ sea importante respecto a Mi.
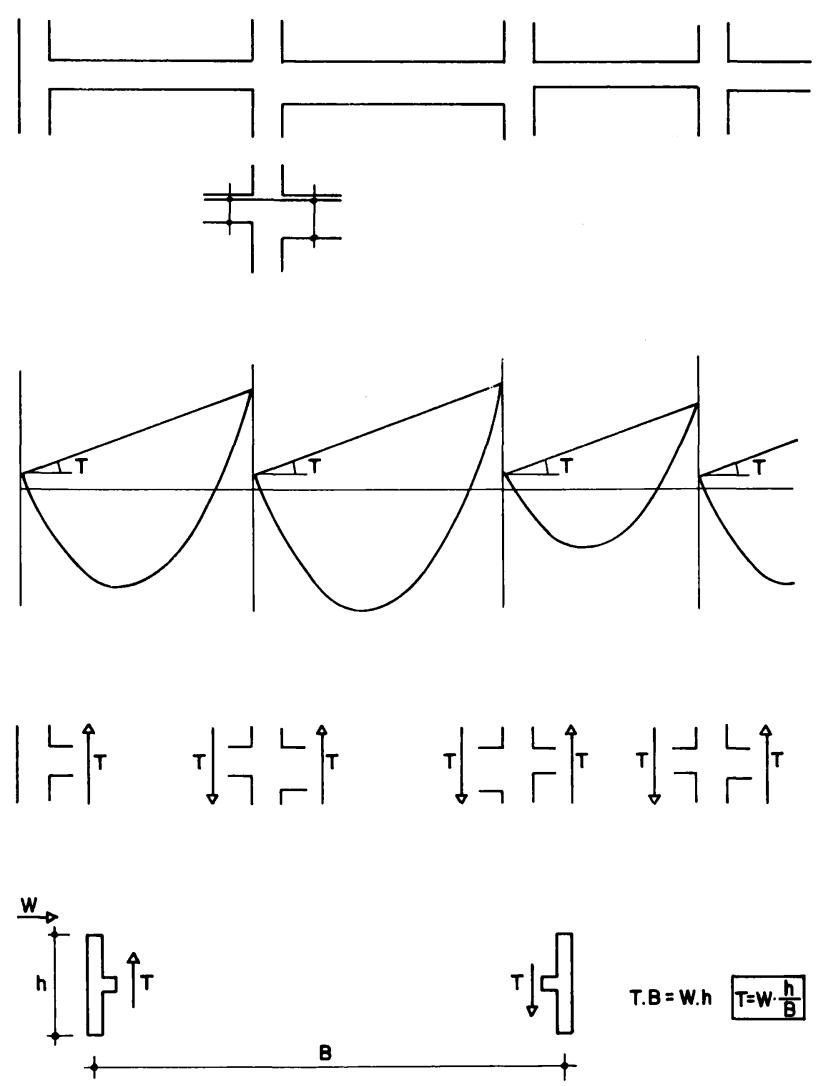

- En cada planta $T \cdot B=W \cdot h \quad T=W \cdot \frac{h}{B}$

- En cada tramo $M w=T \cdot L / 2$

Cuando por razones de diseño general no sea posible variar los cantos de los tramos de la viga, o cuando los $\mathrm{Mw}$ sean pequeños en comparación con los Mi.

La solución más razonable es adoptar un armado igual en todos los tramos, de manera que por una parte se aprovecha la sección de hormigón y por otro se haga contribuir más a resistir la acción horizontal a aquellos tramos más rígidos, aunque ello signifique no optimizar las variaciones de esfuerzo normal en los soportes. 
Planteando el equilibrio de una planta puede obtenerse una fórmula simple para calcular el momento global mínimo $\mathrm{M}$ que debe resistir la viga en función de la geometría, los momentos isostáticos de los tramos para carga vertical y la acción horizontal total en la planta.

$$
\begin{gathered}
M=M i\left(1+\Delta+\frac{\triangle^{2}}{4}\right)=M i\left(1+\frac{\triangle}{2}\right)^{2} \\
\triangle=2\left(\sqrt{\frac{M}{M i}}-1\right)
\end{gathered}
$$

$W \cdot h \leq 2 \Sigma M w=2 \Sigma \quad M i=4 \Sigma \sqrt{M M i}-4 \Sigma M i$
Como $M$ es constante:

$W \cdot h \leq 4 \sqrt{M} \cdot \Sigma \sqrt{M i} \cdot 4 \Sigma M i$

$\sqrt{M} \geq \frac{\frac{W \cdot h}{4}+\Sigma M i}{\Sigma \sqrt{M i}} " M \geq\left(\frac{\frac{W \cdot h}{4}+\Sigma M i}{\Sigma \sqrt{M i}}\right)^{2}$

$\Varangle$ Mi máx

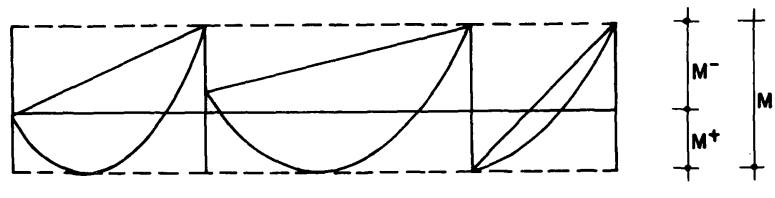

Falta comprobar la compatibilidad de los giros de los distintos tramos y si las rótulas son suficientemente dúctiles, en cada caso concreto.

\section{publicaciones del IETcc / CSIC}

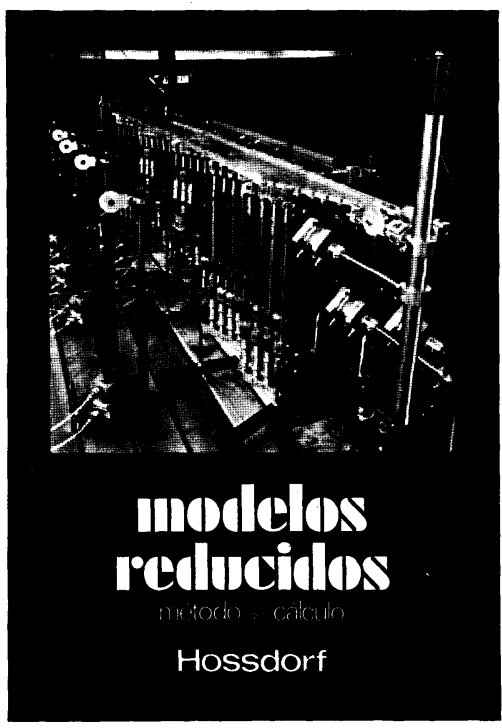

Modelos reducidos. Mótodo de cálculo

H. Hossdorf, Ingeniero Civil

La técnica de los ensayos en modelos reducidos de estructuras sufre hoy día una decisiva metamorfosis. Hasta hace poco era un medio más bien de artesania, que no siempre era tomado en serio por los académicos teorizantes para comprender el comporta miento resistente de las estructuras complejas $y$ a que se acudió las más de las veces, como a un último remedio debido a sus indiscutibles insuficiencias. Sin embargo, en poco tiempo y gracias a su conexión con los ordenadores digitales, se ha transformado en un instrumento cientificamente valioso, que no puede quedar a un lado en la práctica diaria del Ingeniero Proyectista.

Un volumen encuadernado en cartoné plastificado con lomo de tela, de $17 \times 24 \mathrm{~cm}$, compuesto de 250 páginas, 158 figuras $y$ fotografias.

Precios: 1.800 ptas.; \$ USA 26.00

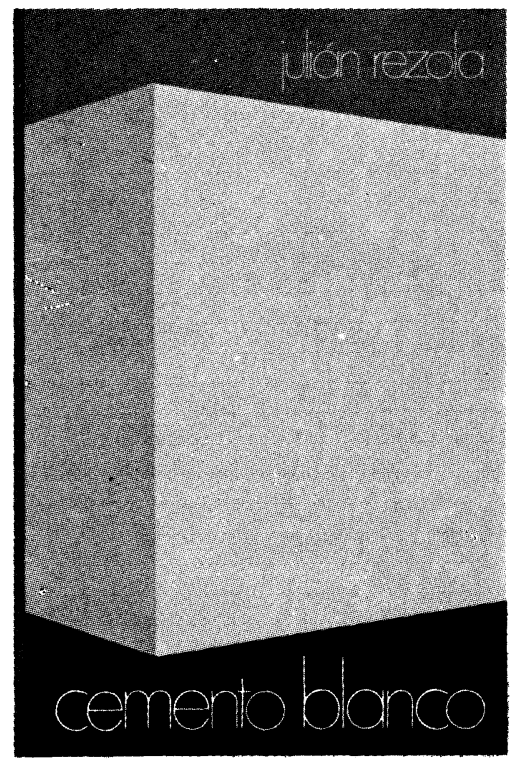

Cemento blanco

Julián Rezola

Ingeniero Quimico Dipl. I. Q. S.

Sabido es que existe una extensa y documentada bibliografia sobre el cemento gris: en cambio, no puede decirse lo mismo acerca del cemento portland blanco, ya que los escritos existentes se refieren tan solo a algunas peculiaridades que le distinguen de aquél.

El autor nos ofrece sus profundos conocimientos y su larga experiencia tanto en laboratorio como en fabricación.

La parte descriptiva del libro se complementa con gráficos, diagramas y fotografias de gran utilidad, destinados a conseguir la aplicación apropiada de este aglomerante.

Un volumen encuadernado en cartoné policerado, de $17,4 \times 24,3 \mathrm{~cm}$, compuesto de 395 páginas, numerosas figuras, tablas y ábacos.

Precios: España, 1.700 ptas.; extranjero, \$ 24.

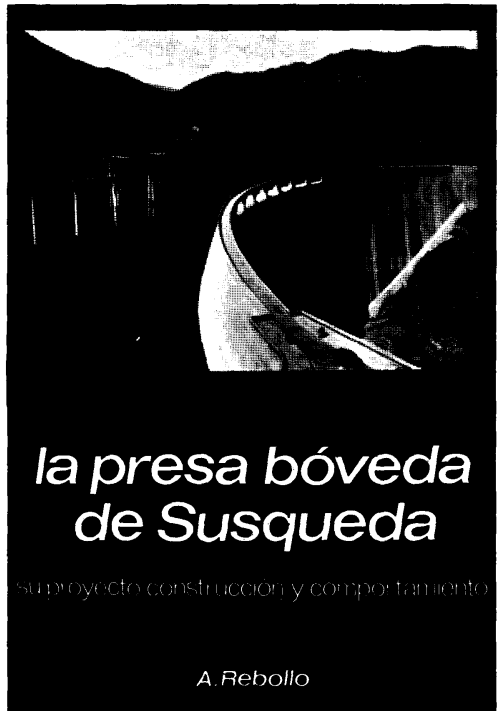

La presa bóveda de Susqueda

A. Rebollo,

Dr. Ingeniero de Caminos

El esfuerzo del constructor de presas se sitúa, por su pretensión de perennidad, a contracorriente de las pren fungible. Pueden evocarse las 10.000 izana por lo fungie. Pueden evocarse las 10.000 gue que gerontológicos para mantener $y$ perfeccionar su perennide En la medida en que todasion de perras, grandes 0 pequeñas, ries, grandes o pequenas, son portadoras de aumentan con las presas es in en de Arturo Rebollo un este torreno marca un cada a Artur Rebollo un este terreno marca un camino a devoción pacos lo que a

Un volumen encuadernado en cartoné plastificado con lomo de ence 408 páginas, 330 figuras y fotografias y 39 tablas.

Precios: 1.700 ptas.; extranjero, \$ USA 24.00 . 